The International Journal of Indian Psychology

ISSN 2348-5396 (e) | ISSN: 2349-3429 (p)

Volume 4, Issue 1, No. 83, DIP: 18.01.165/20160401

ISBN: 978-1-365-64761-1

http://www.ijip.in | October-December, 2016

\title{
Eating Disorders among Psychic Patients: A Clinical Study
}

\author{
Anil Kumar $\operatorname{Jain}^{1 *}$, Sandeep $\operatorname{Kumar}^{2}$
}

\section{ABSTRACT}

Background: Eating disorders (ED) are common conditions that have gained increasing attention over the last two decades. This study was conducted to correlate the eating disorder with body weight dissatisfaction (BWD) and body shape dissatisfaction (BSD) in study population. Materials \& Methods: This study was conducted in department of Psychiatry in year 2012. It was cross sectional type of study conducted on 2360 patients visited the department with any psychiac disorders. Patient information regarding name, age, gender etc was recorded. Body mass index (BMI) was also recorded. Patients were asked to reply the Eating aptitude test (EAT) and BSQ questionnaire. EAT is 26 items evaluated attitudes, feelings and preoccupations in relation to food and weight. The BSQ is a self-report, 34 items questionnaire that evaluates feelings of low self-esteem, the desire to lose weight and body dissatisfaction. For detecting body shape dissatisfaction (BSD), Cooper and Taylor classification was used. The BSD value, $<81$-slight BSD value, 81- 110- moderate BSD and BSD value 111-140- extreme BSD. For detecting BWD, discrepancy between students current and desired weight was considered. Results: Out of 520 patients, 250 (48\%) were males and 270 (51\%) were females. 40\% (208) had BSD, 60\% (312) had BWD, 20\% (104) had positive EAT, 60\% (338) had normal weight, 10 $\%$ (52) had underweight and 25\% (130) had overweight. The magnitudes of BSD in patients with positive EAT. 94 patients (90\%) with a positive EAT expressed BSD. Out of 104 slightly dissatisfied BSD patients, 26 had positive EAT. Of 56 moderately dissatisfied BSD patients, 54 had positive EAT. Of 48 severely dissatisfied BSD patients, 14 had positive EAT. The difference was significant $(\mathrm{P}<0.05)$. Patients with overweight had significantly higher BSD, BWD and positive EAT Conclusion: Eating disorders are increasing day by day. This is an alarming signal for most of the people and westernization is playing important role in this. There is need to educate the people psychologically also.

Keywords: Body Weight Dissatisfaction, Eating Disorders, Eating Aptitude Test

\footnotetext{
${ }^{1}$ Associate Professor, Dept of Psychiatry, Integral Institute of Medical Sciences and Research, Lucknow, Uttar Pradesh, India

${ }^{2}$ Associate Professor, Dept of Psychiatry, Mayo Institute of Medical Sciences, Barabanki, U.P., India

*Responding Author

(C) 2016 Kumar A, Kumar S; licensee IJIP. This is an Open Access Research distributed under the terms of the Creative Commons Attribution License (http://creativecommons.org/licenses/by/2.0), which permits unrestricted use, distribution, and reproduction in any Medium, provided the original work is properly cited.
} 


\section{Eating Disorders among Psychic Patients: A Clinical Study}

Eating disorders (ED) are common conditions that have gained increasing attention over the last two decades. Eating disorders are mental illness defined by abnormal eating habits that negatively affect a person's physical or mental health. Both genetic and environmental factors play important role in causing eating disorder. It has prevalence rate of $1.6 \%$ in females and $0.8 \%$ in males in high-income countries possibly due to cultural and economic factors. Victims of sexual abuse are also likely to develop ED. ${ }^{1}$

Literature shows its association with decreased quality of life, substantial disease burden, depression, substance abuse and suicide. 'Anorexia nervosa' (AN) and 'bulimia nervosa' (BN) are the most important types of eating disorders. American Psychiatric Association Diagnostic and Statistical Manual of Mental Disorders (DSM) also includes 'binge eating disorder' (BED) and 'eating disorder not otherwise specified'. ${ }^{2}$ People with DE also suffer from body dysmorphic disorder (BDD), altering the way a person sees himself or herself. Person diagnosed with BDD also had some type of eating disorder, with $15 \%$ of individuals having anorexia nervosa or bulimia nervosa. The media are oftentimes blamed for the rise in the incidence of eating disorders due to the fact that media images of idealized slim physical shape of people such as model and celebrities motivate or even force people to attempt to achieve slimness themselves. ${ }^{3}$

Socioeconomic status (SES) are among few precipitating factor for eating disorders, presuming that possessing more resources allows for an individual to actively choose to diet and reduce body weight. ${ }^{4}$

Patients in anorexia nervosa refuse to maintain a minimally normal weight, have an intense fear of gaining weight and significant misinterpretation of their body and its shape. Bulimia nervosa is characterized by repeated episodes of binge eating followed by inappropriate behaviour to counteract the calories gained in binges via self-induced vomiting, misuse of laxatives, fasting or excessive exercise. $^{5}$

The body shape is formed of cognitive, perceptual, affective, behavioral and social components while body image is the three dimensional mental representation that each person has of oneself. Body weight dissatisfaction (BWD) and body shape dissatisfaction (BSD) refer to discomfort with one's own body weight and shape and both have been shown to play major roles in the genesis of eating disorders. ${ }^{6}$ This study was conducted to correlate the eating disorder with body weight dissatisfaction (BWD) and body shape dissatisfaction (BSD) in study population.

\section{MATERIALS \& METHODS}

This study was conducted in department of Psychiatry in year 2012. It was cross sectional type of study conducted on 2360 patients visited the department with any psychiac disorders. Patient information regarding name, age, gender etc was recorded. Body mass index (BMI) was also 


\section{Eating Disorders among Psychic Patients: A Clinical Study}

recorded. Patients were asked to reply the Eating aptitude test (EAT) and BSQ questionnaire. ${ }^{7}$ EAT is 26 items evaluated attitudes, feelings and preoccupations in relation to food and weight. The BSQ is a self-report, 34 items questionnaire that evaluates feelings of low self-esteem, the desire to lose weight and body dissatisfaction.

For detecting body shape dissatisfaction (BSD), Cooper and Taylor classification was used. ${ }^{8}$ The BSD value, <81-slight BSD value, 81- 110- moderate BSD and BSD value 111-140- extreme BSD. For detecting BWD, discrepancy between students current and desired weight was considered.

\section{RESULTS}

Table I shows that out of 520 patients, 250 (48\%) were males and 270 (51\%) were females. Graph I shows that 40\% (208) had BSD, 60\% (312) had BWD, 20\% (104) had positive EAT, $60 \%$ (338) had normal weight, $10 \%$ (52) had underweight and 25\% (130) had overweight.

Table II shows the magnitudes of BSD in patients with positive EAT. 94 patients (90\%) with a positive EAT expressed BSD. Out of 104 slightly dissatisfied BSD patients, 26 had positive EAT. Of 56 moderately dissatisfied BSD patients, 54 had positive EAT. Of 48 severely dissatisfied BSD patients, 14 had positive EAT. The difference was significant $(\mathrm{P}<0.05)$. Table III shows that patients with overweight had significantly higher BSD, BWD and positive EAT.

\section{DISCUSSION}

Eating disorders are characterized by extreme emotions, attitudes and behaviours surrounding weights and foods. They are classified into four categories, anorexia nervosa, bulimia nervosa, binge eating disorder and eating disorder not otherwise. These disorders are prevalent, predominantly Western and Westernized cultures with very few reports from underdeveloped and developing countries. This is a psychic disorder seen more prevalent among females as compared to males. ${ }^{9}$

This study was conducted to correlate the eating disorder with body weight dissatisfaction (BWD) and body shape dissatisfaction (BSD) in study population. Body weight dissatisfaction BWD and body shape dissatisfaction (BSD) refer to the discomfort with one's own body weight and shape; both have been shown to play a major role in the genesis of eating disorders. Out of 520 patients, 250 (48\%) were males and 270 (51\%) were females. A study conducted by COX $\mathrm{et}^{10} \mathrm{al}$ in their study revealed that $72 \%$ of females were suffering from Eating disorder.

More patients were concerned about their weight than their shape. Our results are in agreement

with the study done by Haris et al. ${ }^{11}$ The high prevalence of both BWD and BSD is probably due to the rapid societal transition and socio-cultural pressure to achieve an elusive "ideal body". The 


\section{Eating Disorders among Psychic Patients: A Clinical Study}

prevalence of underweight, overweight and normal body weight among our sample is similar to other studies. ${ }^{12,13}$ The prevalence of eating disorder among psychic patients was $22 \%$.

The high prevalence of BSD and BWD in our study is consistent with Lazer et al ${ }^{14}$ finding that $52 \%$ of young women evaluated their shape negatively and 45\% were dissatisfied with their weights. We also tried to establish the relation between BSD, BWD and positive EAT. Patients with overweight had significantly higher BSD, BWD and positive EAT. Our results are in agreement to the results of Sobel J. ${ }^{15}$ However a study conducted by Wong $\mathrm{Y}$ found that overweight patients had higher BWD only.

\section{CONCLUSION}

Eating disorders are increasing day by day. This is an alarming signal for most of the people and westernization is playing important role in this. There is need to educate the people psychologically also.

\section{Acknowledgments}

The author appreciates all those who participated in the study and helped to facilitate the research process.

\section{Conflict of Interests}

The author declared no conflict of interests.

\section{REFERENCES}

1. Oqden CL, Carroll MD, Curtin LR, et al. The prevalence of overweight and obesity in the Unites States. J Am Med Assoc. 2006; 295: 1549-55.

2. Jacobi L, Cash TF. In pursuit of the perfect appearance: discrepancies among self and ideal percepts of multiple physical attributes. J Appl Soc Psychol. 1994; 4: 379-96.

3. Raich RM, Rosen JC, Deus J, et al. Eating disorder symptoms among adolescents in the United States and Spain: a comparative study. Int J Eat Disorder.1992; 11: 63-72.

4. Hudson JI, Hiribi E, Pope HG, Kessler RC. Prevalence and correlates of eating disorders in the National Comorbidity Survey Replication. Biol Psychiatry. 2007; 1: 348-58.

5. World Health Organization. African women: prevalence of eating disorders, global. Geneva, Switzerland: WHO; 1998.

6. Nasser M. Screening for abnormal eating attitudes in a population of Egyptian secondary school girls. Soc Psychiatry Psychiatr Epidemiol. 1994; 29: 25-30.

7. Shuriquie N, Elias T, Abdulhamid M. A study of abnormal eating attitude among Jordanian female college students. Bahrain Medical Bulletin. 1999; 21: 88-90.

8. Cooper PS, Taylor MJ, Cooper Z, Fairburn CG. The development and validation of the body shape questionnaire. Int J Eat Disorder. 1987; 6: 485-94. 


\section{Eating Disorders among Psychic Patients: A Clinical Study}

9. Al-Adawi S, Dorvlo AS, Burke DT. Presence and severity of anorexia and bulimia among male and female Omani and non-Omani adolescents. Am J Acad Child Adolesc Psychiatry. 2002; 41: 1124-30.

10. Cox LM, Lantz CD, Mayhew JL. The role of social physique anxiety and other variables in predicting eating behaviours in college students. Int J Sport Nutr. 1997; 7: 310-7.

11. Harris SM. Body image attitudes and the psychosocial development of college women. $\mathrm{J}$ Psychol. 1995; 129: 315-29.

12. Bowman RL, DeLucia JL. Accuracy of self-reported weight: A meta-analysis. Behav Ther. 1992; 23: 637-55.

13. Cochran WG. Sampling Techniques. New York: John Wiley \& Sons;1977.74-6.

14. Lazer Y, Schatz S. Comprehensive community prevention of disturbed attitudes to weight control: a three-level intervention program. Eating Disorders. J Treat Prev. 1999; 1:3-31.

15. Sobal J. Social influences on body weight. In: KD Brownell \& CG Faiburn (Eds), Eating disorders and obesity: a comprehensive handbook. New York: The Guilford Press; 1995.73-82.

\section{Table I Distribution of patients}

\begin{tabular}{|c|c|c|}
\hline & Total - 520 & \\
\hline Gender & Male & Female \\
\hline Number & $250(48 \%)$ & $270(51 \%)$ \\
\hline
\end{tabular}

Graph I BSD, BWD, Positive EAT, and weight categories among study population

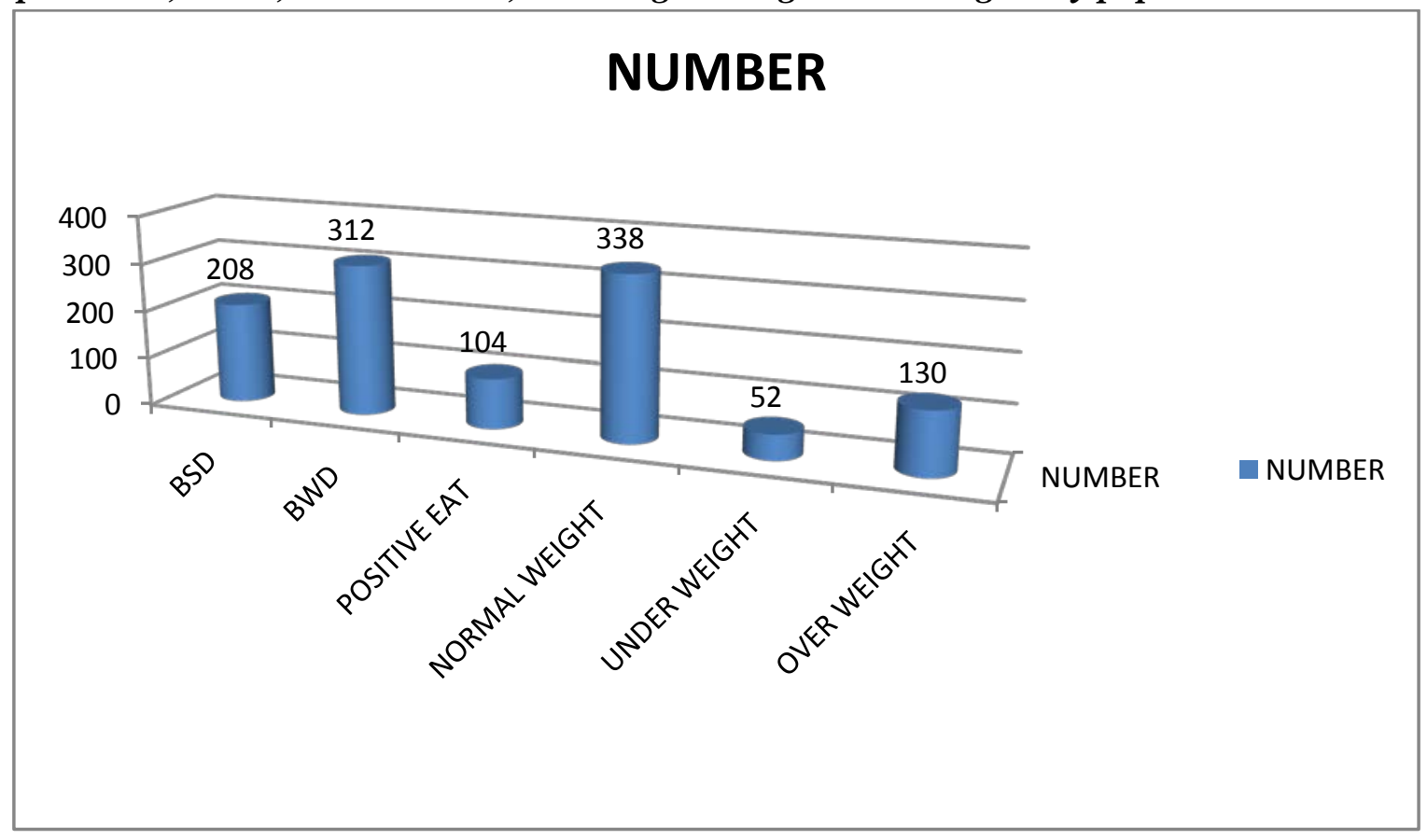

(c) The International Journal of Indian Psychology, ISSN 2348-5396 (e)| ISSN: 2349-3429 (p) | 36 
Eating Disorders among Psychic Patients: A Clinical Study

Table II Magnitude of BSD and positive EAT

\begin{tabular}{|l|c|c|c|}
\hline Level of dissatisfaction & BSD (208) & Positive EAT (104) & P value \\
\hline Slightly dissatisfied & $104(50 \%)$ & $26(25 \%)$ & 0.01 \\
\hline Moderately dissatisfied & $56(27 \%)$ & $54(52 \%)$ & 0.02 \\
\hline Severely dissatisfied & $48(23 \%)$ & $14(13 \%)$ & 0.01 \\
\hline Total & 208 & $94(90 \%)$ & \\
\hline
\end{tabular}

Table III Correlation between BSD, BWD and positive EAT among patients

\begin{tabular}{|l|c|c|c|c|}
\hline & BSD & BWD & Positive EAT & P value \\
\hline Underweight (52) & 11 & 7 & 26 & \\
\hline Normal weight (332) & 73 & 40 & 40 & \\
\hline Over weight (130) & 124 & 265 & 38 & $<0.05$ \\
\hline Total & 208 & 312 & 104 & \\
\hline
\end{tabular}

How to cite this article: Kumar A, Kumar S (2016), Eating Disorders among Psychic Patients: A Clinical Study, International Journal of Indian Psychology, Volume 4, Issue 1, No. 83, ISSN:2348-5396 (e), ISSN:2349-3429 (p), DIP:18.01.165/20160401, ISBN:978-1-365-64761-1 